\title{
Central Tendency Measurement based Unidentified Object Recognition System
}

\author{
${ }^{1}$ S.M. Mohidul Islam, ${ }^{2}$ Abdullah Al Zubaer, ${ }^{3}$ Joyassree Sen, ${ }^{4}$ Bappa Sarkar, ${ }^{5}$ Md. Nazrul Islam \\ ${ }^{1}$ Computer Science and Engineering Discipline, \\ Khulna University, Khulna-9208, Bangladesh \\ 2,3,4,5 Department of Computer Science and Engineering Islamic University, \\ Kushtia-7003, Bangladesh
}

\begin{abstract}
At present era, Object recognition has become one of the most momentous tasks for various applications. The existing approach provides poor and inefficiency result due to complex behavior of real images, object size and shape. For object classification it is very important to extract the exact images from a given dataset. In this case, in many studies we have seen that object recognition has done tremendous representation. We envision a new approach of object recognition based on Mean, Median, Mode, Geometric mean, and standard deviation features using Euclidean distance. The overall goal of my research is to find a robust model for object recognition. The model will be robust in both time and accuracy. The experimental results show that this system can perform comparable recognition of object using these features and the evaluation results conclude that the proposed object recognition system shows better results than many existing methods.
\end{abstract}

Keywords-Object recognition; Central tendency; Standard Deviation; Euclidean distance

\section{INTRODUCTION}

Technology has taken us to a new era of the capability of machines to recognize different objects based on their images [1]. When a human being sees an object, his brain gets some images of the object through his eyes and he can recognize the object easily based on his real life experience [2]. But the task is not easy for a computerized automatic system because the system needs to get images from different angles and it has to have a previous database to compare and contrast. The central tendency measurement based on an unidentified object recognition system can take us a few steps ahead. Though object recognition is a big challenge using computerized object viewing system, the technology has been developed over times. At present object recognition is done getting the images of an object and comparing it with the exact images extracted from the given dataset in the system [6]. In the research, a new approach of object recognition based on mean median geometric mean and standard deviation features using Euclidean distance has been used in object recognition [3]. All these imply a central tendency and that tendency helps us to recognize an unidentified object.

\section{METHODOLOGY AND IMPLEMENTATION}

The system architecture of the research is shown in fig 1. The system involves two phases: training phase and testing phase. Both phase contains some common steps: image acquisition, preprocessing, feature extraction and feature vector creation etc.

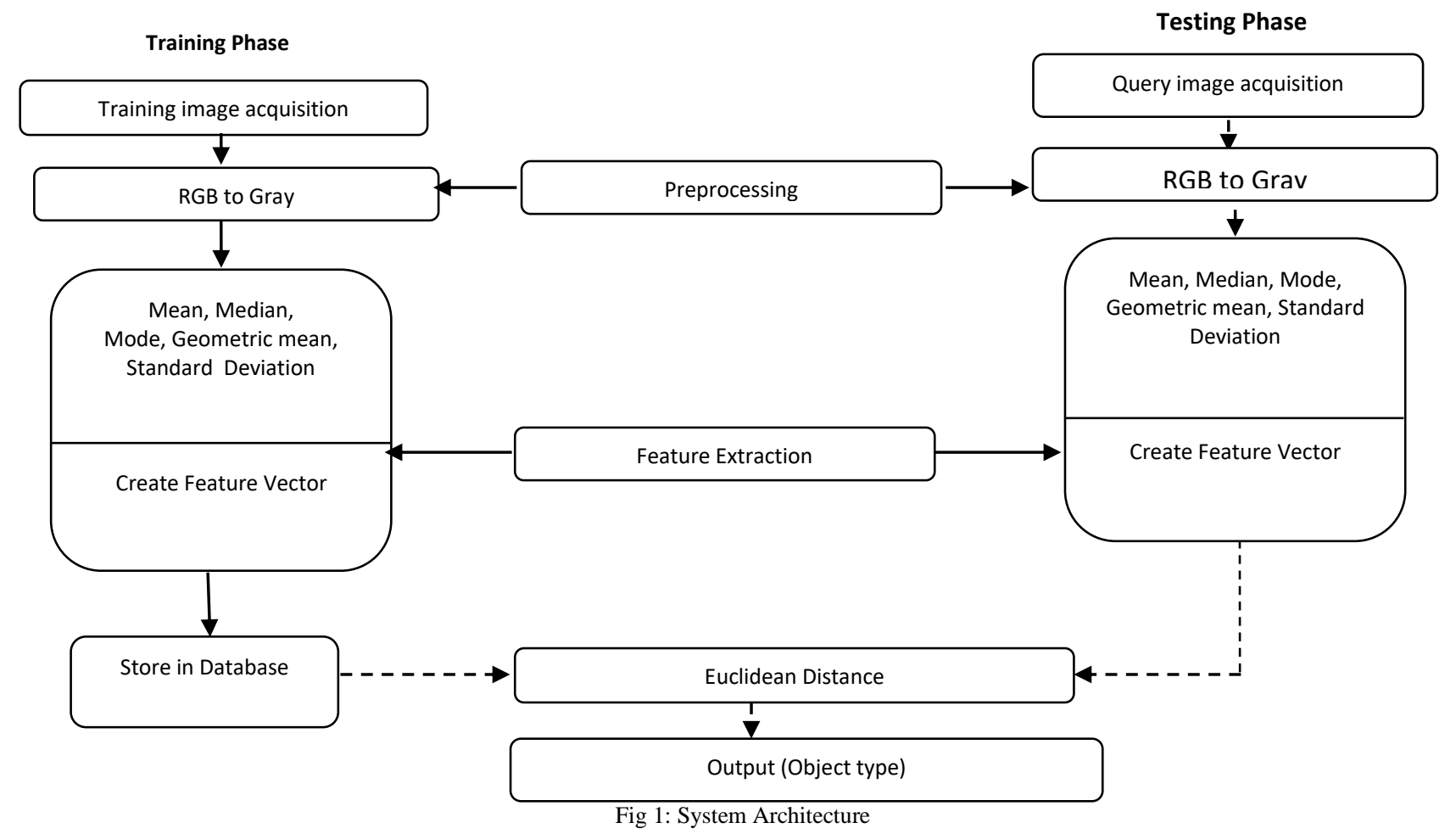




\section{a. Training Phase}

The Steps of the training phases are described in the below:

\section{b. Image Acquisition}

Image of 8 different object types are used for training. All images are resized to $256 \times 256$. Figure 2 shows some sample images of eight different types of objects [7].

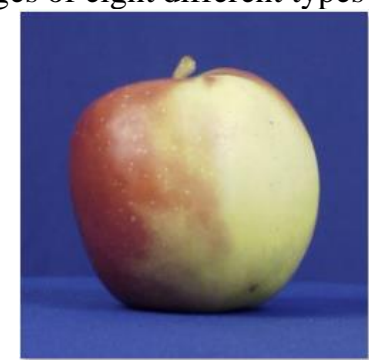

apple1-090-338

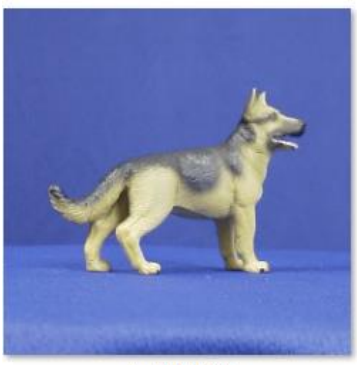

$\operatorname{dog} 1-090-338$

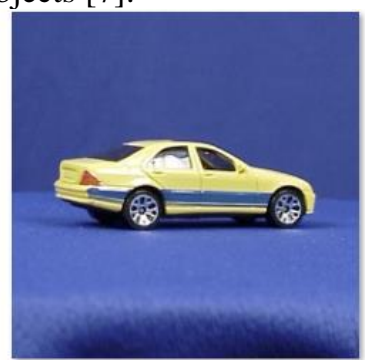

car1-090-338

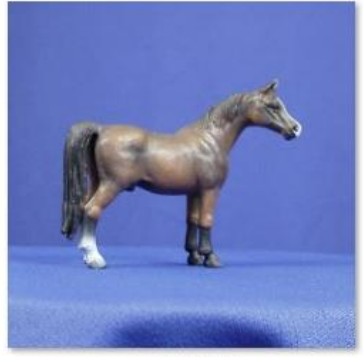

horse1-090-338

Fig 2: Samp

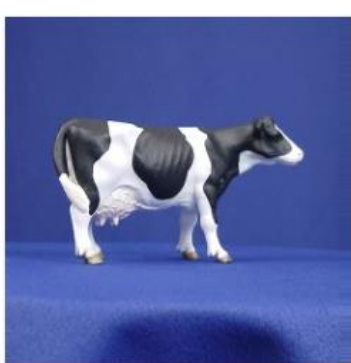

cow1-090-338

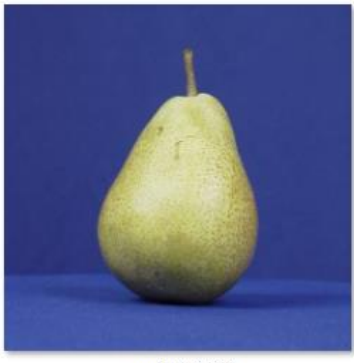

pear1-090-338

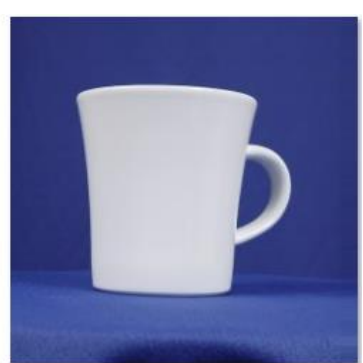

cup1-090-338

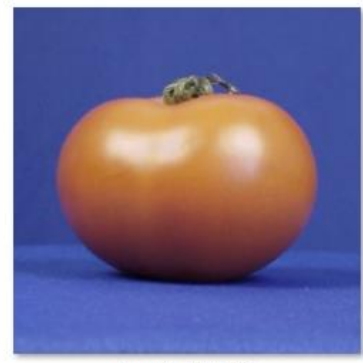

tomato1-090-338

\section{c. Preprocessing}

The images in the dataset are RGB images. The RGB images are converted to gray images. Fig 3 shows a sample gray apple image.

\section{d. Feature Extraction}

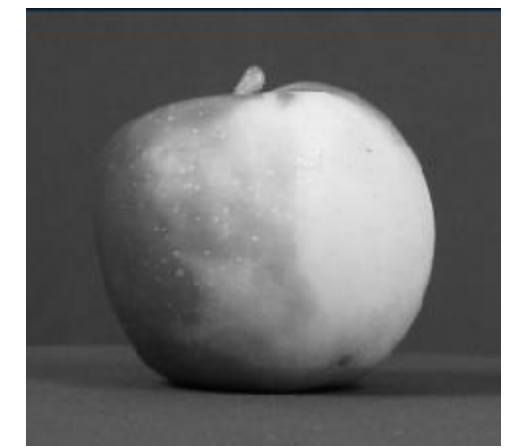

Fig 3: Converted Gray image of apple of fig 2

The features are extracted from processed images. The central tendency meansures such as mean, median, mode, and GM features are used with one data dispersion measure: standard deviation [3].

Measuring the Central Tendency: Mean, Median, Mode, and Geometric mean.

In this section, we look at various ways to measure the central tendency of data. Suppose that we have some attribute $X$, which has been recorded for a set of objects. Let $x_{1}, x_{2}, \ldots \ldots \ldots \ldots, x_{N}$ be the set of $N$ observed values or observations for $X$. Here, these values may also be referred to as the data set (for $X$ ). If we were to plot the observations for $X$, where would most of the values fall? This gives us an idea of the central tendency of the data. Measures of central tendency include the mean, median, mode, and geometric mean.

The most common and effective numeric measure of the "centre" of a set of data is the (arithmetic) mean. Let $\mathrm{x}_{1}, x_{2}$,

be a set of $N$ values or observations, such as for some numeric attribute $X$.

The mean of this set of values is

The median of this set of values is

$$
\bar{x}=\frac{\sum_{i=1}^{N} x_{i}}{N}=\frac{x_{1+} x_{2}+\cdots+x_{N}}{N}
$$

The mode of this set of values is

$$
\mathbf{M e}=\mathbf{L}+\frac{\frac{N}{2}-F_{c}}{f_{m}} \times \boldsymbol{c}
$$

$$
M_{0=L+}+\frac{f_{o-f_{1}}}{2 f_{o}-f_{1}-f_{-1}} \times c
$$


The geometric mean of this set of values is

$$
\mathbf{G}=A n t i-\log \left(\frac{\sum \log x_{i}}{N}\right)
$$

Measuring the Dispersion of Data: Standard Deviation

Variance and standard deviation are measures of data dispersion. They indicate how spread out a data distribution is. A low standard deviation means that the data observations tend to be very close to the mean, while a high standard deviation indicates that the data are spread out over a large range of values [8][9].

The variance of $N$ observations $x_{1}, x_{2}, \ldots \ldots \ldots, x_{N}$ for a numeric attribute $X$ is

$$
\sigma^{2}=\frac{1}{N} \sum_{i=1}^{N}\left(x_{i}-\bar{x}\right)^{2}=\left(\frac{1}{N} \sum_{i=1}^{N} x_{i}^{2}\right)-\bar{x}^{2}
$$

Where, $\overline{\boldsymbol{x}}$ is the mean value of the observations. The standard deviation, $\boldsymbol{\sigma}$, of the observation is the square root of the variance, $\boldsymbol{\sigma}^{2}$.

\section{e. Feature Vector Creation and Storing in Database}

After extracting features from images, the feature vector is created for each image and the feature vectors are stored in the databse [4][5]. Figure 4 shows the sample extracted feature of first 20 images of the dataset. In the figure, $1^{\text {st }}$ column represents

\begin{tabular}{|c|c|c|c|c|c|c|}
\hline 1 & apple & 103 & 71 & 124 & 68 & 56 \\
\hline 2 & apple & 118 & 102 & 128 & 101 & 37 \\
\hline 3 & apple & 116 & 111 & 128 & 91 & 36 \\
\hline 4 & apple & 106 & 85 & 120 & 77 & 46 \\
\hline 5 & apple & 109 & 79 & 123 & 67 & 57 \\
\hline 6 & apple & 111 & 83 & 127 & 79 & 58 \\
\hline 7 & apple & 115 & 101 & 128 & 84 & 46 \\
\hline 8 & apple & 111 & 84 & 126 & 70 & 56 \\
\hline 9 & apple & 116 & 91 & 120 & 69 & 55 \\
\hline 10 & apple & 104 & 72 & 123 & 64 & 55 \\
\hline 11 & car & 97 & 84 & 128 & 83 & 42 \\
\hline 12 & car & 119 & 119 & 128 & 118 & 33 \\
\hline 13 & car & 133 & 131 & 128 & 130 & 33 \\
\hline 14 & car & 117 & 116 & 128 & 116 & 36 \\
\hline 15 & car & 118 & 113 & 128 & 112 & 34 \\
\hline 16 & car & 120 & 117 & 128 & 116 & 33 \\
\hline 17 & car & 111 & 101 & 128 & 99 & 40 \\
\hline 18 & car & 95 & 80 & 128 & 79 & 46 \\
\hline 19 & car & 118 & 119 & 128 & 118 & 35 \\
\hline 20 & car & 108 & 99 & 128 & 97 & 29 \\
\hline
\end{tabular}
the class label, $2^{\text {nd }}$ to $6^{\text {th }}$ column represents the mean, median, mode, standard deviation, and GM features respectively.

Fig 4: Sample extracted features of 20 images (10 apple and 10 car images)

\section{f. Testing Phase: Object Recognition}

The query image acquisition, preprocessing, feature extraction, and fetaure vector vector creations steps of testing phase are same as those of training phase. The distance of the features of the query image from the features of the training images are calculated using Euclidean or L2 distance metric. The class of the image of shortest distance from query image is considered the predicted class of the query image. The most popular distance measure is Euclidean distance (i.e., straight line or "as the crow flies" $)$. Let $i=\left(x_{i} 1, x_{i} 2, \ldots ., x_{i} \mathrm{p}\right)$ and $j=\left(x_{j} 1, x_{j} 2, \ldots, x_{j} \mathrm{p}\right)$ be two objects described by $p$ numeric attributes. The Euclidean distance between objects $i$ and $j$ is defined as

$$
d(i, j)=\sqrt{\left\{\left(x_{i} 1-x_{j} 1\right)^{2}+\left(x_{i} 2-x_{j} 2\right)^{2}+\cdots+\left(x_{i} p-x_{j} p\right)^{2}\right\}}
$$




\section{SIMULATION RESULTS}

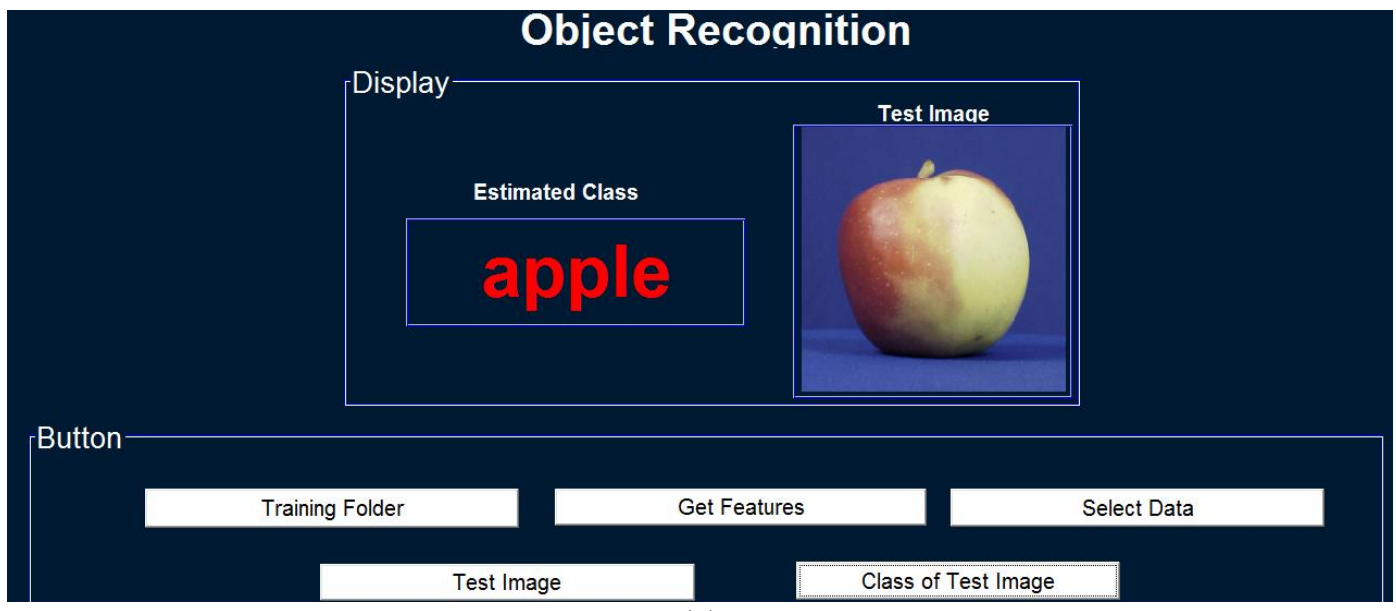

(a)

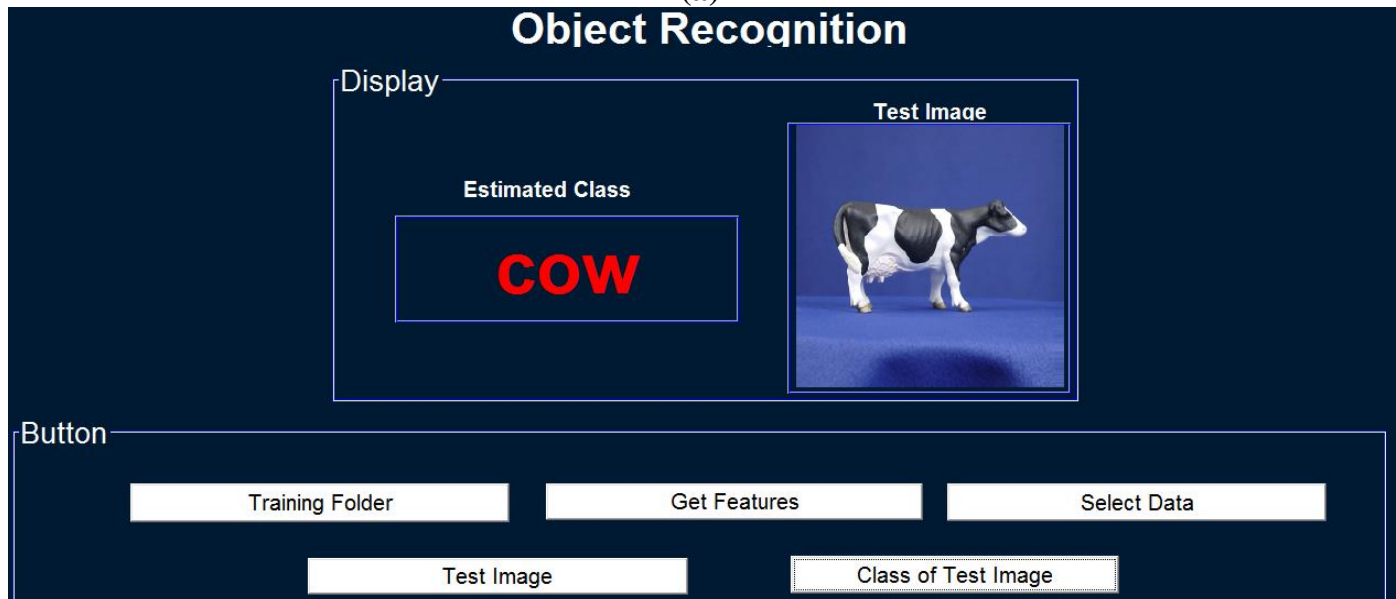

(b)

Fig 5: Sample Object Recognition outputs (apple and cow)

\section{PERFORMANCE ANALYSIS}

The experiments are performed on 80 images. Images include 10 images from 8 different objects (Apple, Car, Cow, Cup, Dog, Horse, Pear, and Tomato). The 10 images of each object vary in angle. Five-fold cross validation is performed, i.e., the images are partitioned into five equal subsets, $D_{1}, D_{2}, D_{3}, D_{4}, D_{5}$. That means, each partition contains 16 images, 2 images from each category. Each time of iteration, one of the partitions is used for testing and other 4 partitions are used for training. This is performed five different times. So each image is tested exactly one time. In each of the iterations, the total number of correctly recognized object is counted and it is $13,15,14,13$, and 13 respectively. So Accuracy of this work is

$$
\text { Accuracy }=((13+15+14+13+13) \times 100 / 80)=85 \% \text {. }
$$

\section{CONCLUSION}

Object recognition from image is an intriguing task in computer vision. It is simple for human being but complex for machine because of complex background, lightening, different viewing angle, size etc. So, a typically automatic and robust system is required to recognize object. In this project, a content-based object recognition system using central tendency measures such as: mean, median, mode, and midrange with one data dispersion measures, standard deviations are discussed. Euclidean or L2 Distance from query image features to training image features are used to find the similar object of the query image. The class of the image object with the shortest distance is considered as the predicted class of the query image. The experimental results show that this system can perform comparable recognition of object using these features and the evaluation results conclude that the proposed object recognition system shows better results than many existing methods.

\section{REFERENCES}

[1] F. Long, H. J. Zhang and D. D. Feng, Fundamentals of Content-basedImage Retrieval, In Multimedia Information Retrieval andManagement, D. Feng Eds, Springer, 2003

[2] A. Goyal and E. Walia, "Variants of Dense Descriptors and Zernike Moments as Features for Accurate Shape-based Image Retrieval," Signal, Image Video Processing, pp. 1-17, 2014.

[3] J. Li and J. Z. Wang. Automatic linguistic indexing of pictures by a statistical modeling approach. IEEE Trans. Pattern Analysis and Machine Intelligence, 25(9):1075-1088, 2003. 
[4] Akio Nagasaka and Yuzuru Tanaka. Automatic video indexing and full-video search for object appearances. In $2^{\text {nd }}$ Working Conference on Visual Database Systems, October 1991

[5] Virginia Ogle and Michael Stonebraker. Chabot: Retrieval from a relational database of images. IEEE Computer, 28(9):40-48, September 1995

[6] Roth, Peter M. \& Winter, Martin (2008). "SURVEYOFAPPEARANCE-BASED METHODS FOR OBJECT RECOGNITION" (PDF). Technical Report. ICG-TR-01/08.

[7] Image Acquisition, https://www.google.com/search?q=image+acquisition\&rlz= 1C1CHBF_enBD813BD813\&oq=Image+Acquisition\&aqs=chrome.0.016.1635j0j4\&source chrome\&ie=UTF-8

[8] McQuarrie, Donald A. (1976). Statistical Mechanics. NY: Harper \& Row. ISBN 0-06-044366-9.

[9] Rothschild, Michael; Stiglitz, Joseph (1970). "Increasing risk I: A definition". Journal of Economic Theory. 2 (3): 225-243. doi:10.1016/00220531(70)90038-4. 